\title{
Sub-MIC levels of purpurin inhibit membrane ATPase-mediated proton efflux activity in the human fungal pathogen Candida albicans
}

\author{
The Journal of Antibiotics (2014) 67, 349-350; doi:10.1038/ja.2013.140; published online 5 February 2014
}

The opportunistic fungal pathogen Candida albicans poses a serious medical threat to human health. This unicellular microbe is part of the normal microbiota on the skin and mucosal surfaces of oral cavity, digestive tract and urogenital system in the patients showing no clinical symptoms. However, in patients with immunosuppression owing to HIV infection, cancer or tissue transplantation, C. albicans can become invasive and cause local and/or disseminated diseases (candidiasis), with high morbidity and mortality rates $(\sim 40-60 \%) .{ }^{1}$ Clinical usefulness of the current limited arsenal of antifungal agents has been hampered by severe side effects, poor pharmacokinetic properties and emergence of multidrug resistance. $^{2}$

Successful colonization and proliferation in host tissues contribute greatly to pathogenicity. One striking virulence trait of C. albicans is its capability to grow and switch between budded yeast and filamentous forms (hyphae). The phenotypic plasticity is indispensable for survival as the pathogenic fungus may experience spatial and temporal variations in different host niches. For example, the human digestive tract can vary from extreme acidity $(\mathrm{pH}<2)$ to alkalinity $(\mathrm{pH}>8)$. Importantly, external $\mathrm{pH}$ determines yeast-to-hypha transition, ${ }^{3}$ and a transient cytoplasmic $\mathrm{pH}$ burst is evident during morphological changes in C. albicans, ${ }^{4}$ suggesting a close physiological-environmental linkage between $\mathrm{pH}$ regulation and pathogenesis.

Membranes are vital for all living cells; not only they serve as selective barriers to the environment, but also define intracellular compartments for diverse and interconnected metabolic processes that include nutrient uptake, ion transport and protein degradation. In particular, $\mathrm{pH}$ homeostasis in C. albicans is tightly controlled by plasma membrane and vacuolar $\mathrm{H}^{+}$-ATPases. ${ }^{5}$ The membrane-bound ATPases generate an electrochemical proton gradient that maintains cytosolic and vacuolar $\mathrm{pH}$ states at the expense of ATP hydrolysis. The functional significance of ATPase activity in C. albicans morphogenesis has been illustrated, as inhibition of the enzyme activity arrested hyphal growth and null mutants were unable to form hyphae and were avirulent. ${ }^{4,6}$ Moreover, a high degree of similarity $(\geqslant 50 \%)$ among fungal ATPases are evident, but they share lesser similarity $(\leqslant 30 \%)$ compared with their mammalian counterparts. ${ }^{7}$ It is thus conceivable that C. albicans membrane-bound ATPases may represent attractive molecular targets in the management of candidiasis through perturbation of intracellular $\mathrm{pH}$ balance and indirect modulation of filamentation.

We have previously demonstrated that purpurin, an anthraquinone pigment commonly found in madder root, possessed potent in vitro anti-Candidal activity, inhibited yeast-to-hypha transition and biofilm development in C. albicans. ${ }^{8,9}$ By virtue of the linkage between morphogenesis and $\mathrm{pH}$ regulation, the present study was designed to investigate the effect of purpurin on the ATPase-mediated proton efflux activity in C. albicans by measuring acidification of external medium. C. albicans SC5314 was obtained from Prof. NAR Gow (University of Aberdeen) and routinely cultured in YPD agar at $30^{\circ} \mathrm{C}$. Purpurin was purchased from TimTec Inc. (Newark, DE, USA) with a purity of $\geqslant 99 \%$. Stock solution $\left(5 \mathrm{mg} \mathrm{ml}^{-1}\right)$ was prepared in distilled dimethyl sulphoxide
(DMSO) and kept at $-20{ }^{\circ} \mathrm{C}$ until use. The final concentration of DMSO was $1 \%$ in all assays. An overnight culture of C. albicans SC5314 was washed twice and resuspended in sterile cold distilled water (to deplete carbon source) at $1 \times 10^{7}$ cells ml ${ }^{-1}$. The cell suspension $(2.7 \mathrm{ml})$ was incubated with different concentrations $\left(0.1-0.5 \mu \mathrm{g} \mathrm{ml}^{-1}\right)$ of purpurin at $30^{\circ} \mathrm{C}$ for $20 \mathrm{~min}$, followed by addition of $20 \%$ glucose $(0.3 \mathrm{ml})$ to induce medium acidification. External $\mathrm{pH}$ was monitored by a $\mathrm{pH}$ meter at 10-min interval for a period of $1 \mathrm{~h}$. Assaying the glucose-induced acidification of external medium is a well-known and convenient method to represent membrane ATPase activity in fungi. ${ }^{5,10}$ Furthermore, to evaluate possible physiological disturbance on purpurin treatment, we measured the growth rates of $C$. albicans in the presence of $3 \mu \mathrm{g} \mathrm{ml}^{-1}$ of purpurin. Fungal cells were grown at $37^{\circ} \mathrm{C}$ with agitation (250 r.p.m.) in YPD broth. Aliquots were withdrawn at $1-\mathrm{h}$ intervals and fungal growth was measured turbidometrically at $600 \mathrm{~nm}$. The assays were performed in triplicate in three different occasions. All data were expressed as mean values with the corresponding standard error of mean (s.e.m.).

A summary of the effect of different concentrations of purpurin on the change of external pH in C. albicans SC5314 is shown in Figure 1. The effect was significant and concentration-dependent. Comparing with the minimum inhibitory concentration $\left(\mathrm{MIC}=5.12 \mu \mathrm{g} \mathrm{ml}^{-1}\right)$ of purpurin against C. albicans, ${ }^{8}$ lower levels of purpurin were able to inhibit glucose-dependent ATPasemediated proton efflux activity and therefore perturbed $\mathrm{pH}$ homeostasis that led to a lesser degree of reduction in external $\mathrm{pH}$. 


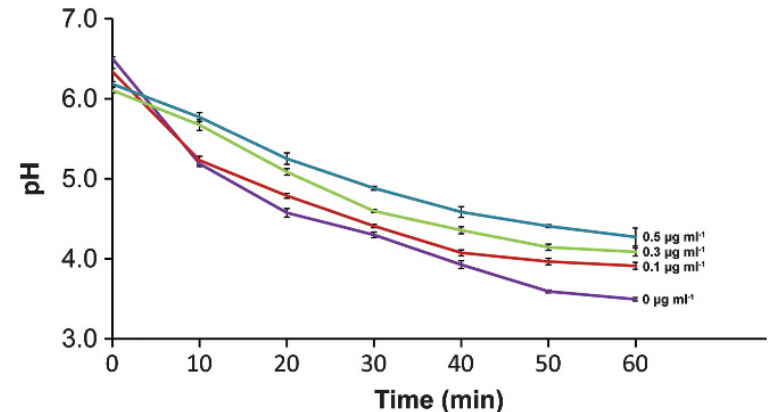

Figure 1 Inhibition of glucose-induced ATPase-mediated proton efflux activity in $C$. albicans by different concentrations of purpurin. Results shown were the average of three independent experiments \pm s.e.m.

Any physiological disturbance of cellular metabolism could be eliminated as the growth of $C$. albicans was not affected even at a higher purpurin concentration of $3 \mu \mathrm{g} \mathrm{ml}^{-1}$ (data not shown). The effective inhibitory effect of sub-MIC levels of purpurin on $C$. albicans ATPases may have clinical relevance, as lower concentrations would reduce the likelihood of the development of drug resistance, especially in combinatorial chemotherapy. To conclude, the present study indicates that sub-MIC levels of purpurin were able to interfere with $\mathrm{pH}$ homeostasis in C. albicans and warrants further investigations.

\section{ACKNOWLEDGEMENTS}

This work was supported by the Research Fund for the Control of Infectious Diseases, the Food and Health Bureau of the Government of the HKSAR (RFCID 11100992); Health and Medical Research Fund, the Food and Health Bureau of the Government of the HKSAR (HMRF 12111292).

Paul Wai-Kei Tsang ${ }^{1}$, Alan Pak-Kin Wong ${ }^{1}$, Han-Sung Jung ${ }^{1}$ and Wing-Ping Fong ${ }^{2}$

${ }^{1}$ Oral BioSciences, Faculty of Dentistry, The University of Hong Kong, Hong Kong, China and ${ }^{2}$ School of Life Sciences,

The Chinese University of Hong Kong, Hong Kong, China E-mail: pwktsang@hku.hk
1 Wenzel, R. P. \& Gennings, C. Bloodstream infections due to Candida species in the intensive care unit: identifying especially high-risk patients to determine prevention strategies. Clin. Infect. Dis. $\mathbf{4 1}$ S389-S393 (2005).

2 Morschhäuser, J. Regulation of multidrug resistance in pathogenic fungi. Fungal Genet. Biol. 47, 94-106 (2010).

3 Davis, D. A. How human pathogenic fungi sense and adapt to $\mathrm{pH}$ : the link to virulence. Curr. Opin. Microbiol. 12, 365-370 (2009).

4 Kaur, S. \& Mishra, P. Differential increase in cytoplasmic $\mathrm{pH}$ at bud and germ tube formation in Candida albicans: studies of a nongerminative variant. Can. J. Microbiol. 40, 720-723 (1994).

5 Shreaz, S. et al. Influences of cinnamic aldehydes on $\mathrm{H}^{+}$extrusion activity and ultrastructure of Candida. J. Med. Microbiol. 62, 232-240 (2013).

6 Shapiro, R. S. \& Cowen, L. E. Uncovering cellular circuitry controlling temperature-dependent fungal morphogenesis. Virulence 3, 400-404 (2012)

7 Perlin, D. S., Seto-Young, D. \& Monk, B.C. The plasma membrane $\mathrm{H}^{+}$-ATPase of fungi: a candidate drug target? Ann. N. Y. Acad. Sci. 834, 609-617 (1997).

8 Kang, K., Fong, W. P. \& Tsang, P. W. K. Novel antifungal activity of purpurin against Candida species in vitro. Med. Mycol. 48, 904-911 (2010).

9 Tsang, P. W. K., Bandara, H. M. H. N. \& Fong, W. P. Purpurin suppresses Candida albicans biofilm formation and hyphal development. PLoS One 7, e50866 (2012).

10 Chan, G., Hardej, D., Santoro, M., Lau-Cam, C. \& Billack, B. Evaluation of the antimicrobial activity of ebselen: role of the yeast plasma membrane $\mathrm{H}^{+}$ -ATPase. J. Biochem. Mol. Toxicol. 21, 252-264 (2007) 